\title{
Markers of Myocardial Ischemia in Patients with Diabetes Mellitus and Severe Obstructive Sleep Apnea - Impact of Continuous Positive Airway Pressure Therapy
}

\author{
Misa Valo ${ }^{1}$, Albert Moller ${ }^{1,2}$ and Claudius Teupe ${ }^{1,2 *}$ \\ ${ }^{1}$ Center of Sleep Medicine, Krankenhaus Sachsenhausen, Frankfurt, Germany \\ ${ }^{2}$ Department of Medicine - Cardiology, Krankenhaus Sachsenhausen, Frankfurt, Germany
}

\begin{abstract}
Introduction: Diabetes Mellitus (T2DM) and obstructive sleep apnea (OSA) are common disorders that often coexist. Both disorders are associated with an increased risk of cardiovascular complications. Repeated nocturnal hypoxia in diabetic patients suffering also from OSA may result in myocardial ischemia. We evaluated whether myocardial ischemia is detectable by ST-segment depression and elevation of high sensitive Troponin T (hs-cTnT) and N-terminal pro B-type natriuretic peptide (NT-proBNP). We also examined the effect of continuous positive airway pressure treatment (CPAP) on these markers.
\end{abstract}

Methods: Fifteen patients with OSA and concomitant T2DM and 26 patients with OSA alone underwent polysomnography at baseline and under CPAP. Blood samples for hs-cTnT and NT-proBNP measurements were drawn prior and immediately after sleep. ST-segment depression was measured at the time of maximum oxygen desaturation during sleep.

Results: The apnea-hypopnea-index and oxygen saturation nadir were similar in both groups. Levels of hscTnT and NT-proBNP did not differ significantly before and after baseline polysomnography and CPAP respectively within the same group. But hs-cTnT levels were significantly higher before and after PSG and CPAP respectively in patients suffering from T2DM and OSA compared to patients with OSA alone. In both groups, we found no significant ST-segment depression at the time of oxygen saturation nadir.

Conclusions: Despite the fact that patients with T2DM and coexisting OSA experienced severe nocturnal hypoxemia we were unable to detect myocardial ischemia or myocyte necrosis evidenced by significant ST-segment depression or elevation of hs-cTnT and NT-proBNP respectively. CPAP had no influence on hs-cTnT and NTproBNP levels.

Keywords: Obstructive sleep apnea; Diabetes mellitus; High sensitive Troponin T; NT-proBNP; Ischemia; Continuous positive airway pressure

\section{Introduction}

Diabetes Mellitus (T2DM) and Obstructive Sleep Apnea (OSA) are common disorders that often coexist [1]. Both, T2DM and OSA are associated with increased cardiovascular morbidity and mortality [2,3]. T2DM is associated with a 2 to 4 -fold increased risk of coronary heart disease [4]. In cross-sectional and case-control studies an increased risk for coronary artery disease and myocardial ischemia in patients with OSA has also been suggested [5,6]. It is possible that the presence of both conditions may result in additive or even synergistic health risks.

OSA is characterized by intermittent hypoxia during sleep, which is associated with elevated sympathetic activity, cardiovascular variability, and intrathoracic pressure changes [7]. Stress imposed on the myocardium by repeated severe hypoxemia during sleep and an increased oxygen demand by sympathetic overstimulation in OSA may result in subclinical myocardial injury [8]. Cardiac troponin $\mathrm{T}$ is an important biomarker in myocardial injury and a predictor of clinical outcome [9]. The cardiac amino-terminal pro-B-type natriuretic peptide (NT-proBNP) is a functional biomarker, that is secreted from the myocytes of the atria and ventricles after myocardial hypoxemia and ventricular volume overload [10]. NT-proBNP production is strongly upregulated in cardiac failure and locally in the area surrounding a myocardial infarction [11].
The standard treatment for moderate and severe OSA is nasal continuous positive airway pressure (CPAP) [12].

We tested the hypothesis that severe OSA may precipitate myocardial ischemia and necrosis in patients with T2DM reflected by ST-segment depression and increased levels of the cardiac markers NT-proBNP and hs-cTnT. As well as testing this hypothesis, we aimed to evaluate whether CPAP therapy can possibly prevent myocardial injury caused by repetitive nocturnal hypoxia.

\section{Materials and Methods}

The prospective study was conducted between February 2012 and September 2013, and was approved by the ethics committee of the State

*Corresponding author: Claudius Teupe, Department of Medicine - Cardiology, Krankenhaus Sachsenhausen - Teaching Hospital of Goethe University Frankfurt Schulstrasse 31, 60594 Frankfurt, Germany, Tel: +49 (0) 696605 1100; Fax: +49 (0) 696605 291100; E-mail: Teupe@em.uni-frankfurt.de

Received December 07, 2014; Accepted January 25, 2015; Published January 30, 2015

Citation: Valo M, Moller A, Teupe C (2015) Markers of Myocardial Ischemia in Patients with Diabetes Mellitus and Severe Obstructive Sleep Apnea - Impact of Continuous Positive Airway Pressure Therapy. J Diabetes Metab 6: 492 doi:10.4172/2155-6156.1000492

Copyright: (c) 2015 Valo M, et al. This is an open-access article distributed under the terms of the Creative Commons Attribution License, which permits unrestricted use, distribution, and reproduction in any medium, provided the original author and source are credited. 
Medical Council of Hessen, Germany (approval number FF 6/2912). Informed consent was obtained from each patient.

\section{Study population}

Patients were screened by polygraphy for the presence of severe OSA with an oxygen desaturation $\leq 80 \%$ during apnea. The study enrolled a group of 15 patients with T2DM and OSA concomitant (group 1) who were referred for Polysomnography (PSG) to the Center of Sleep Medicine at Krankenhaus Sachsenhausen. A group of 26 patients with OSA but without a history of diabetes served as a control (group 2).

Inclusion criteria were as follows: age $>18$ years, severe OSA with an apnea-hypopnea-index (AHI) $\geq 15 /$ h and oxygen desaturation $\leq 80 \%$ at polysomnography, proven history of T2DM (group 1). Exclusion criteria were systolic heart failure (left ventricular ejection fraction $<40 \%$ measured by echocardiography), glomerular filtration rate $<50$ $\mathrm{ml} / \mathrm{min}$ estimated using the Cockcroft-Gault formula.

\section{Polysomnography}

The presence and severity of OSA was determined by overnight complete polysomnography using a computerized system (Alice 5, Philips Respironics, Herrsching, Germany). Standard techniques such as EEG, electrooculography, electromyography, electrocardiogram, thermistor measurements of air flow, thoracoabdominal motion, pulse oximetry of arterial oxyhemoglobin saturation $\left(\mathrm{SPO}_{2}\right)$ and body position were used to monitor sleep disordered breathing. Bedtime was $10 \mathrm{pm}$ to $6 \mathrm{am}$. Sleep stages were scored according to the standard criteria of the American Academy of Sleep Medicine [13]. Apnea was defined as an absence of airflow for $>10 \mathrm{~s}$. Hypopnea was defined as a more than $30 \%$ reduction in airflow accompanied by a decrease in $\mathrm{SPO}_{2}>4 \%$. AHI was calculated as the average number of apneas and hypopneas per hour of sleep. An AHI $\geq 15 / h$ was defined as severe OSA.

\section{Measurement of hs-cTnT and NT-proBNP}

Quantitative measurement of hs-cTnT was achieved via an immunoassay for the in vitro quantitative determination of cardiac troponin $\mathrm{T}$ in human serum and plasma (Cobas e 411 Roche Troponin $\mathrm{T}$ hs STAT; Roche Diagnostics Inc.) with a lower limit of normal $<14$ $\mathrm{pg} / \mathrm{ml}$ representing the 99 th percentile in a normal reference population and a coefficient of variation $<10 \%$. Hs-cTnT values below the Limit of Blank are reported as $<3 \mathrm{ng} / \mathrm{L}$.

NT-proBNP levels were measured with an immunoassay for the in vitro quantitative determination of NT-proBNP in heparinized venous blood (Cobas Roche Cardiac proBNP+; Roche Diagnostics Inc.). Values below the Limit of Blank are reported as $<60 \mathrm{ng} / \mathrm{L}$.

Venous blood samples $(5 \mathrm{ml})$ were collected at different time points: before $(9 \mathrm{pm})$ and after $(7 \mathrm{am})$ polysomnography and CPAP respectively. The measurements were performed immediately after the second set of blood samples were drawn.

\section{ST-segment analysis in the electrocardiogram}

A continuous ECG recording was performed simultaneously during sleep in all patients to screen for ST-segment depression episodes as an indicator of myocardial ischemia. Lead II was used to analyze the ST-segment for myocardial ischemia. Additional leads I, III, aVL, aVR, and aVF were used as backup for further evaluation of features suggestive of cardiac ischemia observed in the main ECG lead. An ischemic episode was defined as a horizontal or downsloping ST-segment depression $\geq 1 \mathrm{~mm}(100 \mu \mathrm{V})$ from baseline, measured 80 ms after the J point. The time point of maximum oxygen desaturation during sleep was determined by continuous oximetry. Ten cardiac cycles after the minimum oxygen saturation the ST-segment changes observed in 10 consecutive cardiac cycles were analyzed and averaged.

\section{Continuous positive air pressure treatment}

Optimal CPAP pressure was titrated in the sleep laboratory on a second night after one diagnostic night at baseline. All patients were monitored under CPAP treatment conducted after pressure titration.

\section{Statistics}

The results are expressed as mean \pm standard deviation (SD) for continuous variables. For the descriptive statistics, the arithmetic mean, standard deviation, median, minimum and maximum, and $1^{\text {st }}$ and $3^{\text {rd }}$ quartiles were calculated. For comparison of the two groups the Fisher exact test and the Wilcoxon-Mann-Whitney U test (U-test) were used. The Wilcoxon Matched Pairs Test was applied for paired samples. Statistical analyses were performed using a statistical software package (BiAS. for Windows, Version 10.05). P-values $<0.05$ were considered statistically significant.

\section{Results}

The study population included a group of 15 patients with T2DM and concomitant OSA (group 1). A group of 26 patients with OSA but without T2DM (group 2) served as a control. Anthropometric characteristics, clinical data, and sleep parameters of both groups are presented in Table 1 . There were no statistically significant differences between the two groups other than the presence of hypercholesterolemia, body mass index and medication with ACE-inhibitors.

In patients who suffered from OSA and T2DM, the mean value for AHI was $57 \pm 18 \mathrm{n} / \mathrm{h}$, the oxygen saturation nadir was $70 \pm 14 \%$, and the maximal duration of sleep-related breathing disorders (SRBD) was $96 \pm 61$ seconds. In the control group, the corresponding values were $48 \pm 21 \mathrm{n} / \mathrm{h}, 72 \pm 13 \%$, and $85 \pm 39$ seconds. The differences between the two groups were not significant.

ST-segment analysis revealed no significant ST depression $(\geq 100$ $\mu \mathrm{V})$ suspicious of myocardial ischemia at the time of the deepest oxygen desaturation, neither in patients who suffered from T2DM and OSA, nor in the control group. ST segment depression was not different in patients with T2DM and OSA compared to patients with OSA alone (Figure 1).

The levels and distribution of the cardiac markers hs-cTnT and NT-proBNP did not differ significantly before and after sleep within the same group. However, hs-cTnT levels were significantly higher before sleep as well as after sleep in patients with T2DM and OSA compared to patients with OSA alone (Figure 2). Hs-cTnT was detectable $(\geq 3 \mathrm{ng} / \mathrm{L})$ in $14(93 \%)$ patients before and in $13(87 \%)$ patients after polysomnography in group 1 . In patients with OSA alone hs-cTnT was detectable in $20(77 \%)$ patients before and in $21(81 \%)$ after polysomnography.

AHI was significantly reduced by CPAP in both patient groups (group 1: AHI $5 \pm 4$, group 2: AHI $5 \pm 5$ ), but showed no effect on hscTnT and NT-proBNP levels (Figure 3).

\section{Discussion}

Despite the fact that patients with T2DM and coexisting OSA experienced severe nocturnal hypoxemia we were unable to detect myocardial ischemia or myocyte necrosis evidenced by significant 


\begin{tabular}{|c|c|c|c|}
\hline & $\begin{array}{c}\text { T2DM and } \\
\text { OSAS } \\
(n=15)\end{array}$ & $\begin{array}{l}\text { OSAS alone } \\
(n=26)\end{array}$ & $p$ value \\
\hline \multicolumn{4}{|l|}{ Demographics } \\
\hline Gender (female/male) & $4 / 11$ & $4 / 22$ & \\
\hline Age (yr) & $58 \pm 9$ & $58 \pm 13$ & n.s \\
\hline Body mass index $\left(\mathrm{kg} / \mathrm{m}^{2}\right)$ & $39 \pm 7$ & $32 \pm 5$ & $<0.01$ \\
\hline \multicolumn{4}{|l|}{ Medical history } \\
\hline Hypertension & $14(93 \%)$ & $18(69 \%)$ & n.s. \\
\hline Hypercholesterolemia & $12(80 \%)$ & $10(38 \%)$ & $<0.05$ \\
\hline Coronary artery disease & $10(67 \%)$ & $11(42 \%)$ & n.s. \\
\hline Arrhythmia & $3(20 \%)$ & $4(15 \%)$ & n.s. \\
\hline Stroke & $2(13 \%)$ & $0(0 \%)$ & n.s. \\
\hline Myocardial infarction & $5(33 \%)$ & $7(27 \%)$ & n.s. \\
\hline COPD & $4(19 \%)$ & $2(10 \%)$ & n.s. \\
\hline \multicolumn{4}{|l|}{ Medication } \\
\hline Insulin & $8(53 \%)$ & - & \\
\hline Oral antidiabetic drugs & $11(73 \%)$ & - & \\
\hline Nitrates & $2(13 \%)$ & $1(4 \%)$ & n.s. \\
\hline$\beta$-blockers & $12(80 \%)$ & $13(50 \%)$ & n.s. \\
\hline Renin inhibitor & $0(0 \%)$ & $1(4 \%)$ & n.s. \\
\hline ACE inhibitors & $10(67 \%)$ & $7(27 \%)$ & $<0.05$ \\
\hline Angiotensin II receptor blockers & $2(13 \%)$ & $6(23 \%)$ & n.s. \\
\hline Diuretics & $8(53 \%)$ & $8(31 \%)$ & n.s. \\
\hline Calcium channel blockers & $6(40 \%)$ & $8(31 \%)$ & n.s. \\
\hline a-blockers & $1(7 \%)$ & $2(8 \%)$ & n.s. \\
\hline \multicolumn{4}{|l|}{ Cardiac and renal function } \\
\hline LVEF (\%) & $60 \pm 8$ & $63 \pm 6$ & n.s. \\
\hline GFR (ml/min) & $144 \pm 48$ & $120 \pm 48$ & n.s. \\
\hline \multicolumn{4}{|l|}{ Polysomnography (baseline) } \\
\hline $\mathrm{AHI}(\mathrm{n} / \mathrm{h})$ & $57 \pm 18$ & $48 \pm 21$ & n.s. \\
\hline Oxygen saturation nadir (\%) & $70 \pm 14$ & $72 \pm 13$ & n.s. \\
\hline
\end{tabular}

Data are presented as mean \pm SD or No (\%). ACE = angiotensin-converting enzyme; $\mathrm{AHI}=$ apnea-hypopnea-index; GFR = glomerular filtration rate; $\mathrm{LVEF}=$ left ventricular ejection fraction

Table 1: Clinical Characteristics and Sleep Parameters.

ST-segment depression or elevation of hs-cTnT and NT-proBNP respectively. CPAP had no influence on hs-cTnT and NT-proBNP levels.

It is well known that OSA is associated with adverse effects on cardiac structure and function. There is a linear relationship between the severity of OSA and patient morbidity and mortality [14]. Cardiac damage in OSA may be caused by activation of the sympathetic nervous system due to hypoxia and changes in negative intrathoracic pressure and increased oxidative stress [15].

We found no abnormal ST-segment depression defined as $>100$ $\mu \mathrm{V}$ according to standard criteria in both groups and no significant difference of ST-segment depression between the two groups measured 10 cardiac cycles after the maximum oxygen desaturation. The mechanism of ST-segment depression during sleep in patients with OSA is not fully understood. Inspiration against occluded upper airways causing periodic negative changes in intrathoracic pressure and alterations in cardiac preload and afterload may result in myocardial ischemia in even the absence of hypoxemia. OSA is often accompanied by hypoxemia and thereby enhances the risk of myocardial ischemia. A previous study by Mooe examined 226 patients with angina pectoris and sleep-disordered breathing [16]. They found a temporal relationship between ST-segment depression and oxygen desaturations only in a minority of $12 \%$ of these symptomatic patients. Other studies

\section{ST segment depression}

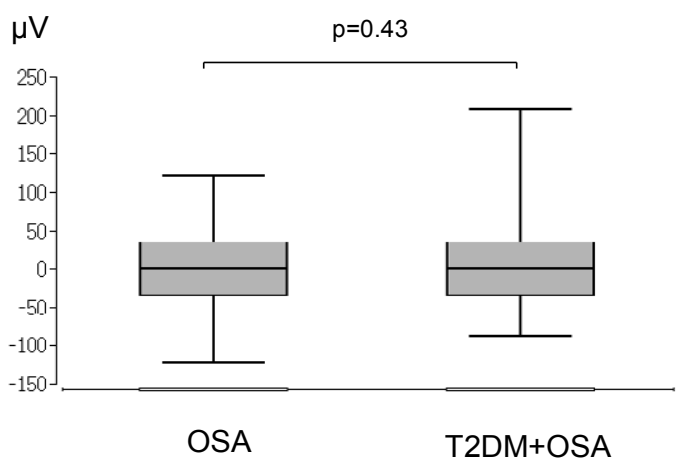

Middle horizontal line inside box indicates median. Bottom and top of the box are 25th and 75th percentiles, and the error bars outside the box represent maximum and minimum values, respectively.

Figure 1: ST-segment depression at the time of the deepest oxygen desaturation CAD - coronary artery disease; OSA - obstructive sleep apnea.

\section{High-sensitive Troponin}

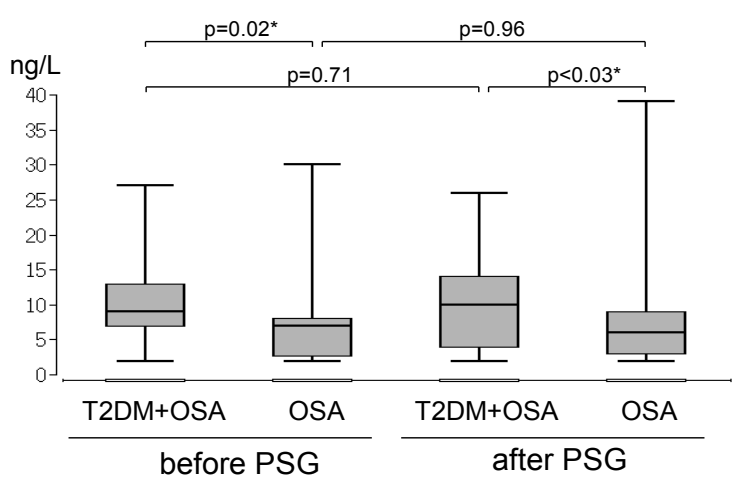

NT-proBNP

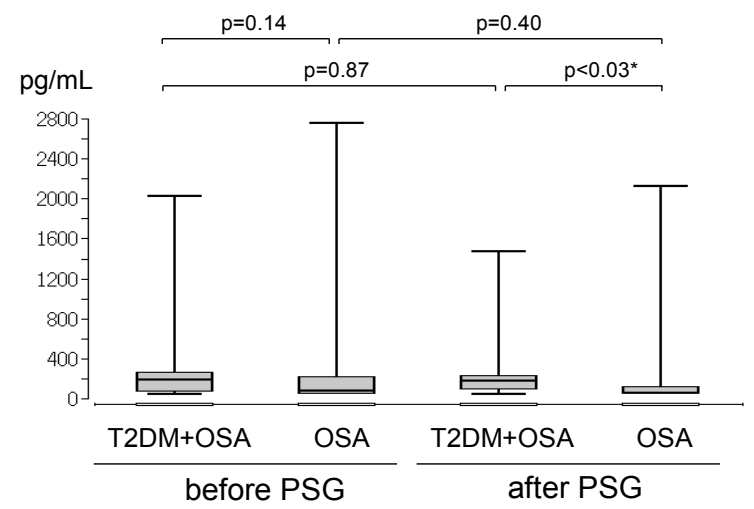

Middle horizontal line inside box indicates median. Bottom and top of the box are 25 th and 75 th percentiles, and the error bars outside the box represent maximum and minimum values, respectively.

Figure 2: Box plots showing high sensitive Troponin T and NT-pro BNP levels before and after polysomnography (PSG) T2DM - type 2 diabetes mellitus, OSA - obstructive sleep apnea. 

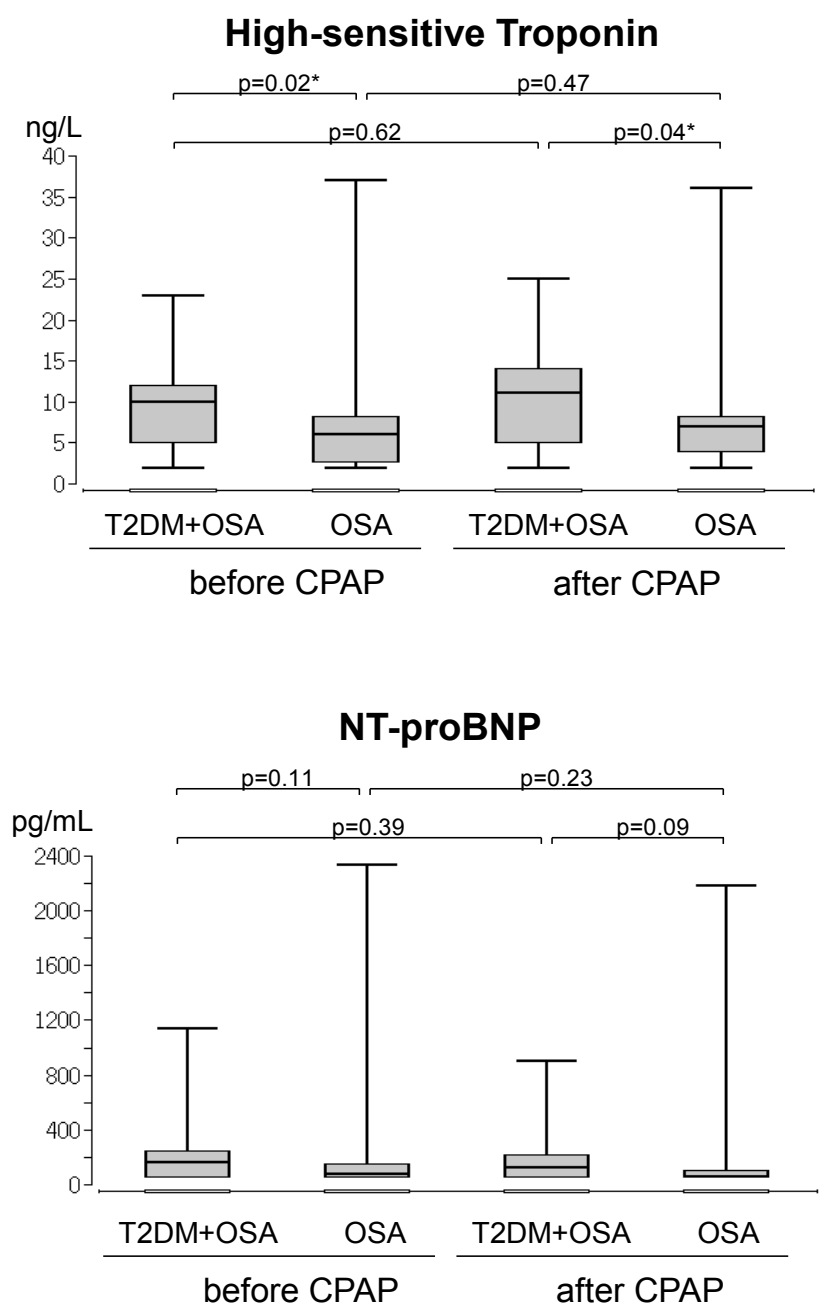

Middle horizontal line inside box indicates median. Bottom and top of the box are 25th and 75 th percentiles, and the error bars outside the box represent maximum and minimum values, respectively.

Figure 3: Box plots showing high sensitive Troponin T and NT-pro BNP levels before and after continuous positive airway pressure (CPAP) T2DM - type 2 diabetes mellitus, OSA - obstructive sleep apnea.

have noted ST-segment depression in about one third of patients with OSA and coexisting coronary artery disease mainly during apneas and reduced oxygen saturation $[17,18]$. Nocturnal ischemia predominantly occurred during the rebreathing phase of the obstructive apneas. In a study with 23 patients, Hanley found asymptomatic ST depression during sleep in $30 \%$ of patients with OSA who did not have a history of coronary artery disease [19]. Interestingly, in this study the duration of sleep, distribution of sleep stages and mean oxygen saturation were not significantly different when ST depression was present or absent.

To our knowledge no other study examined the effect of OSA on hscTnT and NT-proBNP levels in T2DM patients. In the current study, we found no evidence of myocardial ischemia with myocyte necrosis in patients with T2DM and coexisting severe OSA based on a significant increase of hs-cTnT and NT-proBNP levels despite nocturnal hypoxia. Similar to our findings Gami reported no myocardial injury detectable by troponin $\mathrm{T}$ assay in a patient group with coronary artery disease and concomitant OSA [20]. In this study the AHI was comparable to our study but the mean nocturnal oxygen saturation nadir was less distinct $(83 \pm 6 \%)$. In a study including 505 subjects drawn from the general population the proportion of subjects with detectable hs-cTnT increased with increasing severity of OSA. But after adjustment for significant univariate predictors of detectable hs-cTnT, the association between AHI and hs-cTnT was no longer statistically significant [21]. In contrast, Roca found higher hs-cTnT levels independently correlated with OSA severity in middle-aged and older individuals free of coronary heart disease and heart failure [22].

However, hs-cTnT levels were significantly higher before and after sleep in patients who suffered from T2DM and OSA in comparison to patients with OSA alone. Other studies also reported higher troponin levels in diabetic patients but no known coronary artery disease or heart failure. Baseline levels of glycated hemoglobin (HbAlc) were linearly associated with results of hs-cTnT tests [23]. Compared with normoglycemic persons, the adjusted relative risk for incident elevated hs-cTnT is higher in diabetic patients and is associated with a higher risk for future adverse cardiovascular outcome [24-26].

We found no difference in NT-proBNP levels between both groups and no significant change after nocturnal hypoxia. CPAP treatment had no effect on hs-cTnT and NT-proBNP levels in patients without heart failure in both groups despite significant reduction in AHI. In a study by Ljunggren a dose-response relationship between the severity of sleep apnea during the night and the levels of BNP in the morning was seen [27] while other studies found no correlation between OSA severity and NT-proBNP concentration [22,28-30].

The present study did have some limitations that deserve comment. First the number of patients is small due to a high drop out of patients because of insufficient desaturation $(\leq 80 \%)$ at polysomnography. Second it is possible that the chosen time point for analysis of STsegment depression after maximum oxygen desaturation might not represent the time of most severe myocardial ischemia, since other studies suggest that the main cause of ischemia is not an inadequate oxygen supply, but an increase in oxygen demand due to tachycardia and sympathetic activation following the rebreathing phase after an apnea event [18]. We also did not take into account the duration of hypoxemia. Third a single night of CPAP treatment might be too short to have a measureable impact on hs-cTnT and NT-proBNP concentrations in spite of the short half-life of the markers.

\section{Conclusion}

Our study showed that in patients suffering from T2DM and concomitant severe OSA, repeated oxygen desaturation $\leq 80 \%$ did not result in myocardial necrosis based on elevation of hs-cTnT and NTproBNP serum concentrations after sleep. At the time of maximum oxygen desaturation during sleep we found no significant ST-segment depression. Patients with T2DM and concomitant OSA had higher hs-cTnT serum levels than patients with OSA alone, but hs-cTnT concentrations were neither influenced by severe nocturnal hypoxia nor by CPAP. The mechanism of cardiovascular injury in these patients seems to be independent of direct ischemia-induced myocardial necrosis due to nocturnal oxygen desaturation.

\section{Acknowledgements}

We would like to say a special thanks to Simone Rack for her assistance in performing the polysomnography and data acquisition.

\section{References}

1. Kent BD, Grote L, Ryan S, Pepin JL, Bonsignore MR, et al. (2014) Diabetes Mellitus prevalence and control in Sleep Disordered Breathing: the European Sleep Apnea Cohort (ESADA) study. Chest 146: 982-990. 
Citation: Valo M, Moller A, Teupe C (2015) Markers of Myocardial Ischemia in Patients with Diabetes Mellitus and Severe Obstructive Sleep Apnea - Impact of Continuous Positive Airway Pressure Therapy. J Diabetes Metab 6: 492. doi:10.4172/2155-6156.1000492

2. Stamler J, Vaccaro O, Neaton JD, Wentworth D (1993) Diabetes, other risk factors, and 12-yr cardiovascular mortality for men screened in the Multiple Risk Factor Intervention Trial. Diabetes Care 16: 434-444.

3. Won CH, Chun HJ, Chandra SM, Sarinas PS, Chitkara RK, et al. (2013) Severe obstructive sleep apnea increases mortality in patients with ischemic heart disease and myocardial injury. Sleep Breath 17: 85-91.

4. Haffner SM, Lehto S, Ronnemaa T, Pyorala K, Laakso M (1998) Mortality from coronary heart disease in subjects with type 2 diabetes and in nondiabetic subjects with and without prior myocardial infarction. N Engl J Med 339: 229234

5. Peker Y, Kraiczi H, Hedner J, Loth S, Johansson A et al. (1999) An independent association between obstructive sleep apnoea and coronary artery disease. Eur Respir J 14: 179-184.

6. Shahar E, Whitney CW, Redline S, Lee ET, Newman AB, et al. (2001) Sleepdisordered breathing and cardiovascular disease: cross-sectional results of the Sleep Heart Health Study. Am J Respir Crit Care Med 163: 19-25.

7. Bradley TD, Floras JS (2009) Obstructive sleep apnoea and its cardiovascular consequences. Lancet 373: 82-93.

8. van den Aardweg JG, Karemaker JM (1992) Repetitive apneas induce periodic hypertension in normal subjects through hypoxia. J Appl Physiol 72: 821-827.

9. Lindahl B, Toss H, Siegbahn A, Venge P, Wallentin L (2000) Markers of myocardial damage and inflammation in relation to long-term mortality in unstable coronary artery disease. FRISC Study Group. Fragmin during Instability in Coronary Artery Disease. N Engl J Med 343: 1139-1147.

10. Goetze JP, Friis-Hansen L, Rehfeld JF, Nilsson B, Svendsen JH (2006) Atria secretion of B-type natriuretic peptide. Eur Heart J 27: 1648-1650

11. Hall C (2004) Essential biochemistry and physiology of (NT-pro)BNP. Eur J Heart Fail 6: 257-260.

12. Jordan AS, McSharry DG, Malhotra A (2014) Adult obstructive sleep apnoea Lancet 383: 736-747.

13. Iber C A-IS, Chesson A, Quan S (2007) The AASM Manual for the Scoring of Sleep and Associated Events: Rules, Terminology and Technical Specification. (1stedtn). American Academy of Sleep Medicine, Westchester, IL. USA.

14. Shivalkar B, Van de Heyning C, Kerremans M, Rinkevich D, Verbraecken J et al. (2006) Obstructive sleep apnea syndrome: more insights on structural and functional cardiac alterations, and the effects of treatment with continuous positive airway pressure. J Am Coll Cardiol 47: 1433-1439.

15. McNicholas WT, Bonsigore MR (2007) Sleep apnoea as an independent risk factor for cardiovascular disease: current evidence, basic mechanisms and research priorities. Eur Respir J 29: 156-178.

16. Mooe T, Franklin KA, Wiklund U, Rabben T, Holmstrom K (2000) Sleepdisordered breathing and myocardial ischemia in patients with coronary artery disease. Chest 117: 1597-1602.
17. Schafer H, Koehler U, Ploch T, Peter JH (1997) Sleep-related myocardia ischemia and sleep structure in patients with obstructive sleep apnea and coronary heart disease. Chest 111: 387-393.

18. Peled N, Abinader EG, Pillar G, Sharif D, Lavie P (1999) Nocturnal ischemic events in patients with obstructive sleep apnea syndrome and ischemic heart disease: effects of continuous positive air pressure treatment. J Am Coll Cardiol 34: 1744-1749.

19. Hanly P, Sasson Z, Zuberi N, Lunn K (1993) ST-segment depression during sleep in obstructive sleep apnea. Am J Cardiol 71: 1341-1345.

20. Gami AS, Svatikova A, Wolk R, Olson EJ, Duenwald CJ, et al. (2004) Cardiac troponin T in obstructive sleep apnea. Chest 125: 2097-2100.

21. Randby A, Namtvedt SK, Einvik G, Hrubos-Strom H, Hagve TA, et al. (2012) Obstructive sleep apnea is associated with increased high-sensitivity cardiac troponin T levels. Chest 142: 639-646.

22. Querejeta RG, Redline S, Punjabi N, Claggett B, Ballantyne CM, et al. (2013) Sleep apnea is associated with subclinical myocardial injury in the community The ARIC-SHHS study. Am J Respir Crit Care Med 188: 1460-1465.

23. Rubin J, Matsushita K, Ballantyne CM, Hoogeveen R, Coresh J, et al. (2012) Chronic hyperglycemia and subclinical myocardial injury. J Am Coll Cardiol 59: 484-489.

24. Selvin E, Lazo M, Chen Y, Shen L, Rubin J, et al. (2014) Diabetes mellitus, prediabetes, and incidence of subclinical myocardial damage. Circulation 130: 1374-1382.

25. Eubanks A, Raza F, Alkhouli M, Glenn AN, Homko C, et al. (2012) Clinica significance of troponin elevations in acute decompensated diabetes without clinical acute coronary syndrome. Cardiovasc Diabetol 11: 154.

26. Hillis GS, Welsh P, Chalmers J, Perkovic V, Chow CK, et al. (2014) The relative and combined ability of high-sensitivity cardiac troponin $\mathrm{T}$ and $\mathrm{N}$-terminal proB-type natriuretic peptide to predict cardiovascular events and death in patients with type 2 diabetes. Diabetes Care 37: 295-303.

27. Ljunggren M, Lindahl B, Theorell-Haglow J, Lindberg E (2012) Association between obstructive sleep apnea and elevated levels of type B natriuretic peptide in a community-based sample of women. Sleep 35: 1521-1527.

28. Hubner RH, El Mokhtari NE, Freitag S, Rausche T, Goder R, et al. (2008) NTproBNP is not elevated in patients with obstructive sleep apnoea. Respir Med 102: $134-142$.

29. Cifci N, Uyar M, Elbek O, Suyur H, Ekinci E (2010) Impact of CPAP treatment on cardiac biomarkers and pro-BNP in obstructive sleep apnea syndrome. Sleep Breath 14: 241-244.

30. Gottlieb JD, Schwartz AR, Marshall J, Ouyang P, Kern L, et al. (2009) Hypoxia, not the frequency of sleep apnea, induces acute hemodynamic stress in patients with chronic heart failure. J Am Coll Cardiol 54: 1706-1712. 